\title{
Phenotypic and Genotypic Characterization of Mycobacteria Isolates from Buruli Ulcer Suspected Patients Reveals the Involvement of Several Mycobacteria in Chronic Skin Lesions
}

\author{
Aka Nguetta ${ }^{1, *}$, Coulibaly $\mathrm{N}^{1}$, Kouamé-Elogne N $\mathrm{C}^{1}$, Acquah K J R ${ }^{1}$, Amon Aby Christiane ${ }^{1}$, \\ Kouadio Kouamé ${ }^{1}$, Konan N'Guessan ${ }^{3}$, Koffi Aboa ${ }^{2}$, Kadio M.C ${ }^{2}$, Yao Aubin ${ }^{3}$, \\ Paul Saunderson ${ }^{4}$, Kakou-Ngazoa $\mathrm{ES}^{1}$, Faye-Ketté ${ }^{1}$, H et Dosso $\mathrm{M}^{1}$ \\ ${ }^{1}$ Department of Research, Pasteur Institute, Abidjan, Côte d'Ivoire \\ ${ }^{2}$ Department of Health, Buruli Ulcer National Program, Abidjan, Côte d'Ivoire \\ ${ }^{3}$ MAP International, National Office, Abidjan, Côte d'Ivoire \\ ${ }^{4}$ Head Office, American Leprosy Missions, Greenville, USA \\ *Corresponding author: aka_nguetta@yahoo.fr
}

\begin{abstract}
Buruli ulcer is a cutaneous mycobacterial disease that occurs in tropical countries in sub-Saharan Africa, South-East Asia, Australia and America. The responsible pathogen is Mycobacterium ulcerans. Côte d'Ivoire is the most affected country, with more than 30 endemic health districts reporting a large number of chronic skin lesions. The clinical forms and the severity of ulcers vary from one patient to another. Samples from suspected patients were analyzed by PCR at Pasteur Institute of Côte d'Ivoire, as recommended by WHO. IS2404 sequence was detected in $61 \%$ of cases, incriminating M. ulcerans in chronic cutaneous lesions. For the other cases the etiology was not identified, thus raising several questions. Are all reported "Buruli ulcer" cases really caused by M. ulcerans? Would other mycobacteria be involved in the occurrence of chronic skin lesions considered as "Buruli ulcer"? BU suspected patients were enrolled in endemic areas of Côte d'Ivoire. Samples were collected from cutaneous lesions and transported to the lab at $+4^{\circ} \mathrm{C}$ in $2 \mathrm{ml}$ of Middlebrook $7 \mathrm{H} 9$ medium supplemented by Cetylpiridium chloride. The centrifugation pellet was taken with saline buffer to perform microscopic examination and mycobacteria isolation on Lowenstein-Jensen medium. Biochemical characteristics were described by the nicotinic acid detection according to Konno protocol, by the Nitrates reduction test, by the catalase activity detection at $22^{\circ}$ and $68^{\circ} \mathrm{C}$ and the Wayne's Tween 80 hydrolysis test. Genotypic characteristics were determined by PCR with $1 \mathrm{ml}$ of bacterial suspension targeting the insertion sequences (IS6110, IS2404, and IS2606), the plasmid virulence genes and Miru-VNTR loci (Miru-1, VNTR 6, VNTR 19, ST-1). A total of 47 mycobacterial strains were isolated with 3 different types of colonies whose microscopic examination showed Acid-Alcohol-Resistant Bacilli. $65.9 \%$ of isolates expressed biochemical characters in favor of M. ulcerans strains and $6.4 \%$ in favor of M. marinum strains. For $29.8 \%$ of isolates, the characteristics were related to atypical mycobacterial species. The genotyping targeting the IS6110, IS2606 and IS2404 insertion sequences allowed simultaneous amplification in 53.2\% of isolates. IS2404 was amplified in $93.6 \%$ of isolates; IS6110 was amplified in $74.5 \%$ of isolates and IS2606 was amplified in $70.2 \%$ of isolates. Five genotypes were identified corresponding to various species of mycobacteria: genotypes 1 and 2 accounted for $63.8 \%$ with all the 3 insertion sequences and biochemical characteristics in favor of $M$. ulcerans strains; genotype 4 accounted for $6.4 \%$ of isolates with insertion sequences and biochemical characteristics in favor of $M$. marinum strains; The strains of genotypes 3 and 5 expressed molecular and biochemical characters relating to various non-M. ulcerans mycobacteria. Virulence genes were found in $72.3 \%$ of isolates corresponding to $90 \%$ of $M$. ulcerans strains and $60.7 \%$ of non-M. ulcerans mycobacteria. This study confirmed the involvement of several genotypes of M. ulcerans and other mycobacteria in chronic cutaneous lesions suspected as cases of Buruli ulcer in Côte d'Ivoire.
\end{abstract}

Keywords: Buruli ulcer, M. ulcerans, insertion sequences, other mycobacteria, virulence genes

Cite This Article: Aka Nguetta, Coulibaly N D, Kouamé-Elogne N C, Acquah K J R, Amon Aby Christiane, Kouadio Kouamé, Konan N’Guessan, Koffi Aboa, Kadio M.C, Yao Aubin, Paul Saunderson, Kakou-Ngazoa ES, Faye-Ketté, and H et Dosso M, "Phenotypic and Genotypic Characterization of Mycobacteria Isolates from Buruli Ulcer Suspected Patients Reveals the Involvement of Several Mycobacteria in Chronic Skin Lesions.” American Journal of Microbiological Research, vol. 6, no. 3 (2018): 79-87. doi: 10.12691/ajmr-6-3-3. 


\section{Introduction}

Buruli ulcer (BU) is a cutaneous mycobacterial disease that occurs in thirty tropical countries, especially in sub-Saharan Africa [1,2,3,4,5], South-East Asia [6,7,8,9], Australia [10,11,12] and Latin America [13,14,15]. The responsible pathogen of the disease is Mycobacterium ulcerans, a slow-growing mycobacterium. Côte d'Ivoire is one of the most affected countries, with more than 30 endemic health districts whose have been reporting for many years a large number of chronic skin lesions attributed rightly or wrongly to $M$. ulcerans [4]. The evolution of M. ulcerans infection occurs in two successive stages: a pre-ulcerative phase marked by the appearance of a nodule, a plaque or an œdema. The pre-ulcerative forms and the severity of ulcers vary from one patient to another. Some patients have small lesions that are easy to cure while others have large ulcers for a long time. Biological confirmation is routinely performed by Polymerase Chain Reaction (PCR) methods targeting the IS2404 insertion sequence and ketoreductase (KR) in clinical samples, as recommended by WHO [16]. Several samples of BU suspected patients were analyzed by the National Buruli Ulcer Reference Center of Pasteur Institute of Côte d'Ivoire. For several years, the molecular diagnosis results incriminated at most M. ulcerans in $61 \%$ of those samples [4,17]. For the other cases (39\%), the etiology was not identified, thus raising several questions. Are all reported BU cases really caused by $M$. ulcerans? Would other mycobacteria be involved in the occurrence of chronic skin lesions liked observed in BU? Would there be a genetic diversity of M. ulcerans strains in relation to the clinical polymorphism observed in endemic areas? These questions remained for a long time unanswered, prompted the study of several isolated strains of BU suspected patients in several endemic areas of Côte d'Ivoire. The aim of this work was to study the bacteriological, biochemical and molecular characteristics of isolated mycobacterial strains from cutaneous lesions considered as cases of M. ulcerans infection.

\section{Patients and Methods}

\subsection{Patient's Enrollment and Mycobacterial Isolation}

BU suspected cases (patients with nodule, plaque, œdema or ulcer) were enrolled in nine endemic areas of Côte d'Ivoire. Epidemiological and clinical informations were collected using UB 01, as recommended by WHO. Samples consisted of exudates or fine needle aspiration fluid [18] were discharged into a tube containing $2 \mathrm{ml}$ of Middlebrook 7H9 medium supplemented by Cetylpiridium chloride $(0.5 \%)$ and transported to the lab at $+4^{\circ} \mathrm{C}$. $10 \mathrm{ml}$ of sterile water were added to the samples previously placed in a 15 $\mathrm{ml}$ Falcon tube. The mixture was centrifuged at $3000 \mathrm{rpm}$ for 20 minutes

The pellet was resuspended in $1 \mathrm{ml}$ of saline buffer ( $\mathrm{NaCl}, 9 \%$ ) to inoculate four tubes of Löwenstein-Jensen medium. The tubes were incubated at $32^{\circ} \mathrm{C}$ and a daily observation was instituted till the appearance of colonies [19].

\subsection{Phenotypic and Molecular Characterization of Strains}

Several tests have been performed to describe the phenotypic and genotypic characteristics of isolates. The cultural characteristics were described based on the timing of colony appearance, the pigment secretion, and the morphology of colonies. Microscopic examination to detect Acid-fast bacilli was performed by using dried smears made with $5 \mu \mathrm{l}$ of bacterial suspension and staining by the Ziehl-Neelsen coloration [20,21]. Biochemical characteristics were described by the Nicotinic Acid detection according to Konno protocol (Niacin-test), by the Nitrates reduction test, by the catalase activity detection at $22^{\circ}$ and $68^{\circ} \mathrm{C}$, and the Wayne's Tween 80 hydrolysis test $[1,21]$. The genotypic characteristics were determined by molecular tests using $1 \mathrm{ml}$ of bacterial suspension. The strains were first characterized using insertion sequences (IS6110, IS2404, IS2606) secondly the plasmid virulence factor coding gene (Ketoreductase, $\mathrm{KR}$ ) and then a VNTR typing. The insertion sequence IS6110 is common to the genus Mycobacterium wilton et cousin 1992 [22] while the insertion sequences IS2404 and IS2606 [23] are carried by M. ulcerans's genome respectively in about 250 and 100 copies. The third tests targeted minisatellite markers: MIRU-VNTR (Mycobateria Interpersed Repeated Units- Variable Number of Tandem Repeated). The using method is as described by Stragier and several authors [24,25,26,27]. A set of four markers have been used: MIRU1, ST1, VNTR6 and VNTR19. Those markers permitted to distinguish M.ulcerans from other mycolactone producing Mycobacteria (MPM) and M.ulcerans like- strains (M. marinum, M. Pseudoshotsii).

PCR were performed in a 9700 PCR system Thermocycler (Applied Biosystems, USA) [28,29,30,31,32]. The IS6110, IS2404, IS2606 and ketoreductase (KR) were amplified according to the following program: a pre-denaturation phase at $94^{\circ} \mathrm{C}$ for 2 minutes, followed by 35cycles consisted of a denaturation phase at $94^{\circ} \mathrm{C}$ for 1 minute, an annealing at $66^{\circ} \mathrm{C}$ for 1 minute and extension phase at $72^{\circ} \mathrm{C}$ for 1 minute. A final extension at $72^{\circ} \mathrm{C}$ for 5 minutes stopped the reaction. PCR products were revealed on a $1.5 \%$ gel electrophoresis containing Sybr Green. A 100 bp molecular weight marker was used to evaluate PCR products size. For Genotyping, the thermocycler (GeneAmp 9700) was programmed as following: an initial denaturation for $5 \mathrm{~min}$ at $95^{\circ} \mathrm{C}$, followed by 40 cycles of $94^{\circ} \mathrm{C}$ for $1 \mathrm{~min}$, $58^{\circ} \mathrm{C}$ for 1 min (MIRU-1, locus 6, VNTR 19) or $65^{\circ} \mathrm{C}$ (for ST-1) and $72^{\circ} \mathrm{C}$ for $1 \mathrm{~min}$. DNA inhibition was tested by serial dilution of the DNA extract. Positive DNA samples of $\mathrm{M}$. ulcerans and $\mathrm{M}$. marinum from the Institute of Tropical Medicine were tested for each amplification.

The GoTaq G2 Flexi DNA polymerase kit (Promega Corporation, USA) was used for the PCR mixes containing $0.2 \mu \mathrm{M}$ of each primer, $1.5 \mu \mathrm{M}, \mathrm{MgCl}, 0.1 \mu \mathrm{M}$ dNTPs, 1 unit Taq polymerase, $1 \mathrm{X}$ of buffer and $3 \mu$ l of DNA template for a final volume of $50 \mu 1$. The revelation was made on a GelDoc Bioanalyzer (BioRad) after electrophoresis.

The size of the amplicons was used to determine the copy number of the targeted sequence and the profile result was given in code form characterizing one type of polymorphism [25,26]. 
Table 1. Methods, targets and primer's sequences used for molecular characterization

\begin{tabular}{|c|c|c|c|c|}
\hline Targets & Primers & Direct and indirect Sequences & Amplicons size (pb) & References \\
\hline \multirow{2}{*}{ Mu5/6 } & \multirow{2}{*}{ IS2404 } & GAT CAA GCG TTC ACG AGT GA & \multirow{2}{*}{600} & \multirow{2}{*}{ Ross et al., 1997} \\
\hline & & GGC AGT TAC TTC ACT GCA CA & & \\
\hline \multirow{2}{*}{$\mathrm{Mu} / / 8$} & \multirow{2}{*}{ IS2606 } & CCG TCA CAG ACC AGG AAG AAG & \multirow{2}{*}{332} & \multirow{2}{*}{ Stinear et al, 1999} \\
\hline & & TGC TGA CGG AGT TGA AAA ACC & & \\
\hline \multirow{3}{*}{ Mycgen-F/R } & \multirow{2}{*}{ IS6110 } & AGA GTT TGA TCC TGG CTC AG & \multirow{2}{*}{1030} & \multirow{2}{*}{ Wilton et Cousin (1992) } \\
\hline & & TGC ACA CAG GCC ACA AGG GA & & \\
\hline & \multirow{3}{*}{ PKS } & TCA CGG CCT GCG ATA TCA & & \multirow{3}{*}{ Fyfe et al., 2007} \\
\hline \multirow[t]{2}{*}{ KR-F/R } & & TTG TGT GGG CAC TGA ATT GAC & & \\
\hline & & GGC TTG ACT CAT GTC ACG TAA G & & \\
\hline \multirow{2}{*}{ MIRU1 F/R } & \multirow{2}{*}{ MIRU1 } & GCT GGT TCA TGC GTG GAA G & & \multirow{8}{*}{ Stragier et al., 2004} \\
\hline & & GCC CTC GGG AAT GTG GTT & & \\
\hline \multirow{2}{*}{ VNTR6 F/R } & \multirow{2}{*}{ LOCUS 6} & GAC CGT CAT GTC GTT CGA TCC TAG T & & \\
\hline & & GAC ATC GAA GAG GTG TGC CGT CT & & \\
\hline \multirow{2}{*}{ VNTR19 F/R } & \multirow{2}{*}{ LOCUS 19} & CCG ACG GAT GAA TCT GTA GGT & & \\
\hline & & TGG CGA CGA TCG AGT CTC & & \\
\hline \multirow{2}{*}{ ST1 F/R } & \multirow{2}{*}{ ST1 } & CTG AGG GGA TTT CAC GAC CAG & & \\
\hline & & CGC CAC CCG CGG ACA CAG TCG & & \\
\hline
\end{tabular}

Table 2. Data from different reference strains used in the study

\begin{tabular}{ccccccccc}
\hline Reference strains & Origin & IS2404 & IS2606 & KR & Miru-1 & ST-1 & Locus 6 & Locus 19 \\
\hline ITM 9540 & Australia (Queensland) & Positive & Positive (300bp) & Positive & Positive (400bp) & Negative & Positive (400bp) & Positive (350bp) \\
\hline ITM 94-0821 & Côte d'Ivoire & Positive & Positive & Positive & Positive (500bp) & Positive (400bp) & Positive (400bp) & Positive (380bp) \\
\hline ITM 98-912 & China & Positive & Positive & Positive & Positive (400bp) & Positive (400bp) & Positive (500bp) & Positive (500bp) \\
\hline ITM 842 & Suriname & Positive & Positive & Positive & Negative & Positive (400bp) & Positive (480bp) & Positive (400bp) \\
\hline ITM 97-483 & Ghana & Positive & Positive & Positive & Positive (500bp) & Positive (400bp) & Positive (500bp) & Positive (350bp) \\
\hline ITM 99-0006 & Australia (Queensland) & Positive & Positive & Positive & Positive (500bp) & Positive (400bp) & Positive (500bp) & Positive (400bp) \\
\hline
\end{tabular}

The data of these methods applied to the reference strains are recorded in Table 2.

\section{Results}

\subsection{Microbiological and Biochemical Characteristics of Isolates}

47 isolates were obtained on Löwenstein-Jensen medium from 9 endemic areas. Microscopic examination showed Acid-Alcohol-Resistant Bacilli. Three types of colonies were described among the isolates. The first type accounted for $65 \%$ of the isolates and consisted of firm, rounded, bulging, yellow-gold colonies. The colonies appeared after 3 to 6 weeks of incubation at $32^{\circ} \mathrm{C}$ (Figure $1 \mathrm{~A}$ ). The second type accounted for $25 \%$ of the isolates and consisted of rough, irregular-shaped, cauliflower-like, whitish, brittlelike colonies. The colonies appeared between 1 and 3 weeks of incubation at $32^{\circ} \mathrm{C}$ (Figure 1B). The third type of culture represented $10 \%$ of the isolates and associated rough, whitish, cauliflower-like colonies and rough yellow-gold colonies with curved surfaces. The colonies appeared after 3 weeks of incubation at $32^{\circ} \mathrm{C}$ (Figure 1C).

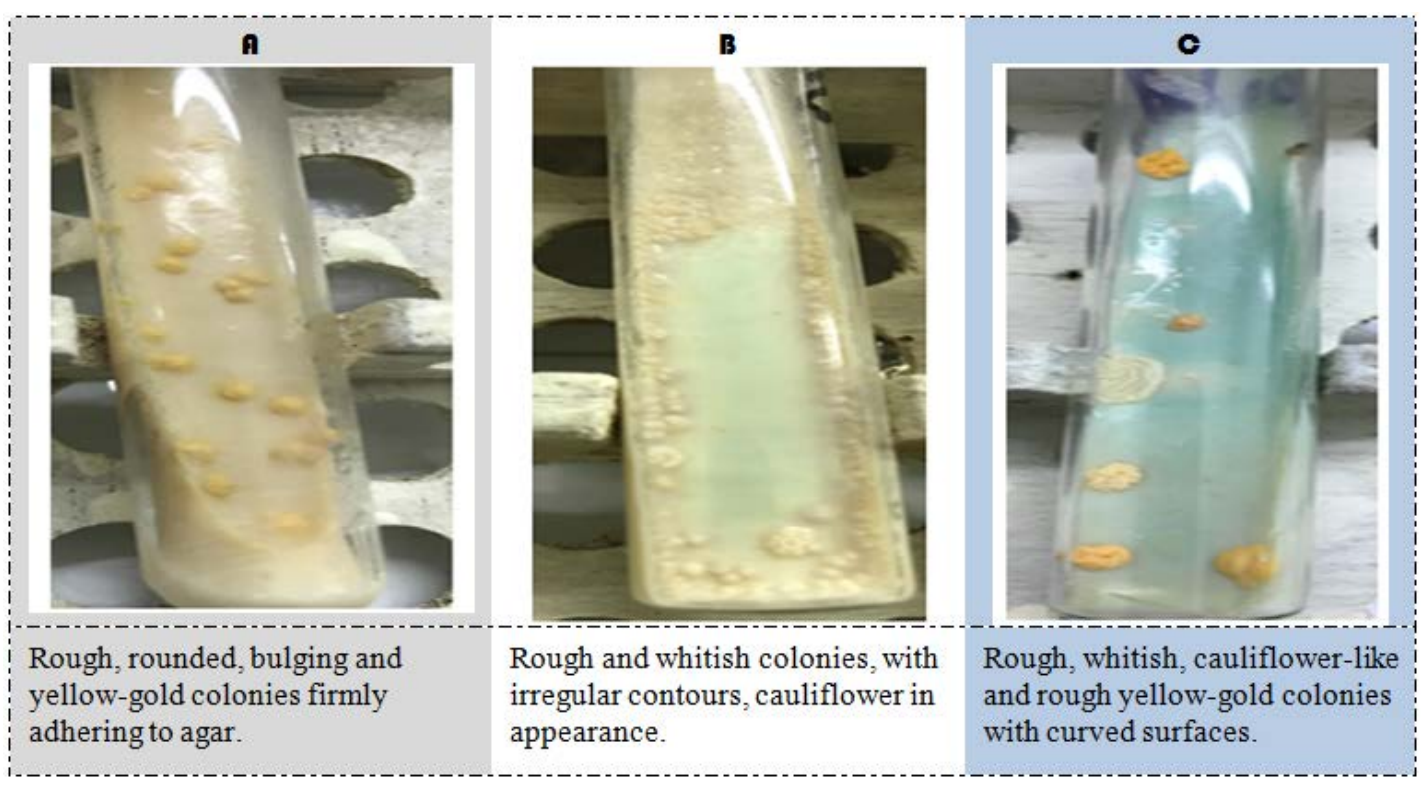

Figure 1. Phenotypic characteristics of bacterial colonies described on Löwenstein-Jensen medium 


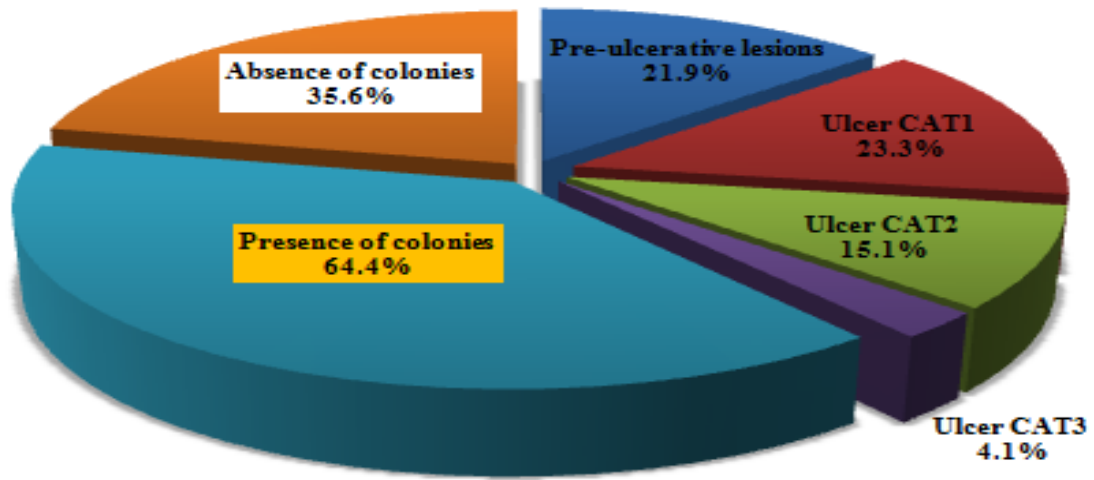

Figure 2. Performance of Mycobacteria isolation on Löwenstein-Jensen medium

Table 3. Microbiological, biochemical and molecular characteristics of mycobacterial isolates

\begin{tabular}{|c|c|c|c|c|c|c|c|c|c|c|c|}
\hline Endemic areas & Isolates $(\mathrm{N}=47)$ & $\mathrm{C} 22$ & C68 & NP & NR & HT80 & PSA+ & IS6110 & IS2606 & IS2404 & Mycobacteria Species \\
\hline \multirow{2}{*}{ Tonkpi } & 4 & + & - & - & - & - & + & + & - & + & M. smegmatis \\
\hline & 5 & + & + & - & - & - & + & + & + & + & M. ulcerans \\
\hline \multirow{2}{*}{ Lôh Djiboua } & 2 & + & - & + & - & - & + & - & - & + & M. chelonae \\
\hline & 1 & + & + & - & - & - & + & + & + & + & M. ulcerans \\
\hline \multirow{4}{*}{ Mé } & 1 & + & - & + & - & - & + & - & - & + & M. chelonae \\
\hline & 2 & + & + & - & - & - & + & + & + & + & M. ulcerans \\
\hline & 1 & + & + & - & - & - & + & - & + & + & M. ulcerans \\
\hline & 1 & + & + & + & - & - & + & + & + & - & M. marinum \\
\hline \multirow{3}{*}{ Gbêkê } & 2 & + & - & - & - & - & + & - & + & + & M. ulcerans \\
\hline & 3 & + & + & - & - & - & + & + & + & + & M. ulcerans \\
\hline & 1 & + & + & - & + & + & + & + & - & + & M. smegmatis \\
\hline \multirow{3}{*}{ Marahoué } & 2 & + & + & - & - & - & + & + & - & + & M. smegmatis \\
\hline & 1 & + & - & - & - & - & + & + & + & + & M. ulcerans \\
\hline & 1 & + & + & - & - & - & + & - & + & + & M. ulcerans \\
\hline \multirow{2}{*}{ Bélier } & 2 & + & + & + & - & - & + & + & + & - & M. marinum \\
\hline & 5 & + & - & - & - & - & + & + & + & + & M. ulcerans \\
\hline \multirow{3}{*}{ Agnéby-Tiassa } & 2 & + & + & + & - & - & + & - & - & + & M. chelonae \\
\hline & 2 & + & - & - & - & - & + & + & + & + & M. ulcerans \\
\hline & 1 & + & + & - & - & - & + & - & + & + & M. ulcerans \\
\hline Sud-Comoé & 3 & + & - & - & - & - & + & + & + & + & M. ulcerans \\
\hline \multirow{2}{*}{ Gôh } & 2 & + & + & + & - & - & + & - & - & + & M. chelonae \\
\hline & 3 & + & - & - & - & - & + & + & + & + & M. ulcerans \\
\hline
\end{tabular}

C22 : Catalase at $22^{\circ} \mathrm{C}$; C68: Catalase at $68^{\circ} \mathrm{C}$; NP : Nicotinic acid Production ; NR : Nitrate reductase; HT80: Hydrolysis of Tween 80 ; PSA+: Growth on medium with Para amino Salicylic Acid.

30 strains of M. ulcerans (63.8\%); 3 strains of M. marinum (6.4\%); 7 strains of M. chelonae (14.9\%); 7 strains of M. smegmatis (14.9\%).

The rate of mycobacteria isolation on Löwenstein-Jensen medium was $64.4 \%$. The majority of isolates (45.2\%) were obtained with samples collected on pre-ulcerative lesions and ulcer of category 1. Mycobacterial isolation was lower in ulcers of categories 2 and 3, with rates of $15.1 \%$ and $4.1 \%$, respectively. In $35.6 \%$ of samples from BU suspected patients, no mycobacteria were isolated after 12 weeks incubation at $32^{\circ} \mathrm{C}$ (Figure 2).

Three groups of isolates were identified based on biochemical characters detected. Most of isolates (31/47) expressed biochemical characters in favor of a $M$. ulcerans strain. In $6.4 \%$ of the isolates (3/47), the biochemical characteristics were in favor of a $M$. marinum strain. For $27.6 \%$ of the isolates (13/47), the biochemical characteristics were related to atypical mycobacterial species (Table 3).

\subsection{Molecular Characteristics of the Isolates}

Molecular characterization of mycobacteria with the inserted elements IS6110, IS2606 and IS2404 allowed simultaneous amplification in 53.2\% of the isolates (Table 3). Specifically, IS2404 was amplified in $93.6 \%$ of the isolates (44/47); IS6110 was amplified in $74.5 \%$ of isolates (35/47) and IS2606 was amplified in $70.2 \%$ of isolates (33/47). Depending on the number of amplified insertion sequences and biochemical characters expressed, five different genotypes were identified corresponding to various species of mycobacteria (Table 3). The strains of genotype 1 accounted for $53 \%$ of the isolates that possessed the three insertion sequences and biochemical characteristics in favor of M. ulcerans strains. Genotype 2 is composed of $10.6 \%$ of the isolates (5/47) with the IS2606 and IS2404 insertion sequences and expressed biochemical characteristics of $M$. ulcerans strains. Both genotypes 1 and 2 accounted for $63.8 \%$ of isolates (30/47) from suspicious lesions of Buruli ulcer. Genotype 4 composed of strains with sequences IS6110 and IS2606 accounted for 6.4\% of the isolates. Their microbiological and biochemical characteristics were identical to those of $M$. marinum strains. Genotype 3 accounted for $14.9 \%$ of isolates (7/47) 
and had sequences IS6110 and IS2404. Genotype 5 also accounted for $14.9 \%$ of the isolates (7/47) and had only IS2404 sequence. However, both genotypes 3 and 5 expressed biochemical characters relating to various strains of mycobacteria. The association of microbiological, biochemical and genotypic characters allowed the identification of $M$. chelonae and $M$. smegmatis species isolated (Figure 3).

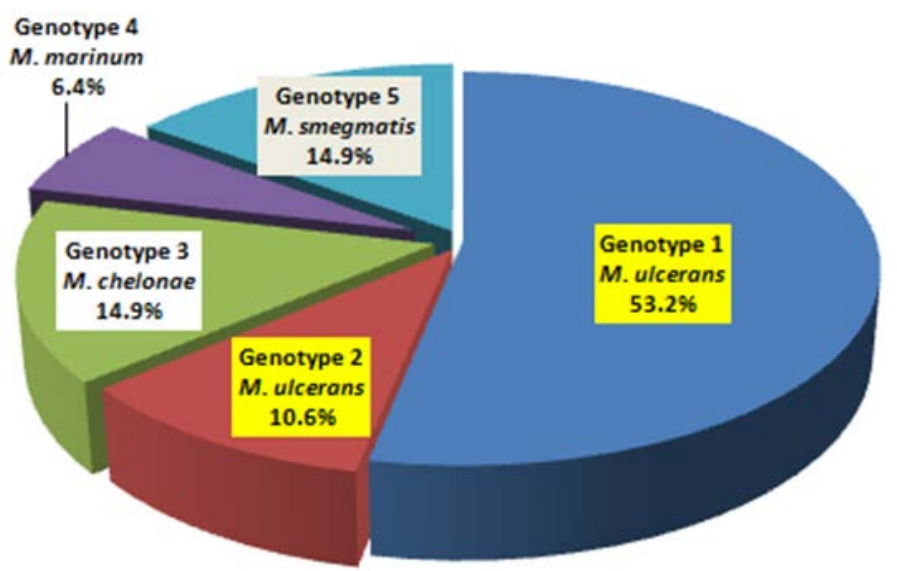

Figure 3. Genotypes identified by targeting IS6110, IS2404 and IS2606 insertion sequences

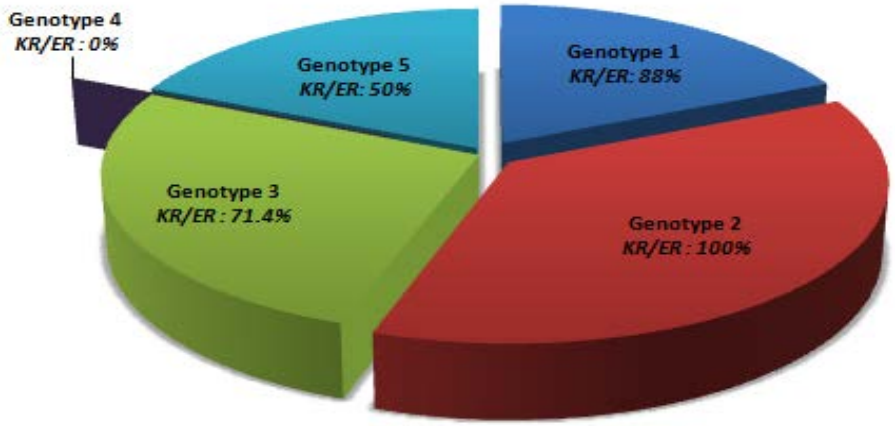

Figure 4. Mycobacterial strains possessing virulence genes among genotypes

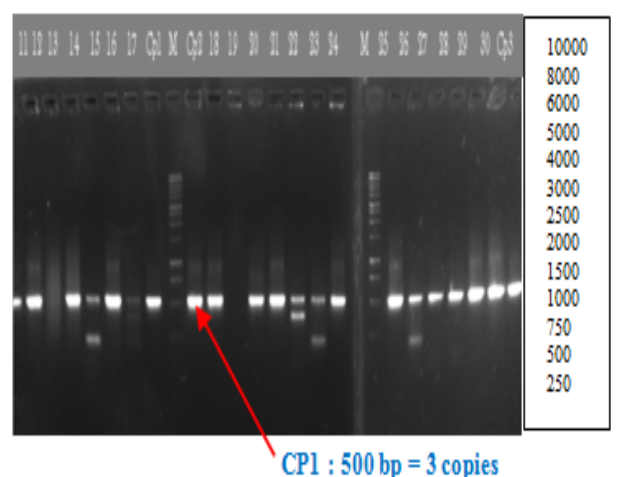

ค. VNTR MU / Locus Miru-1

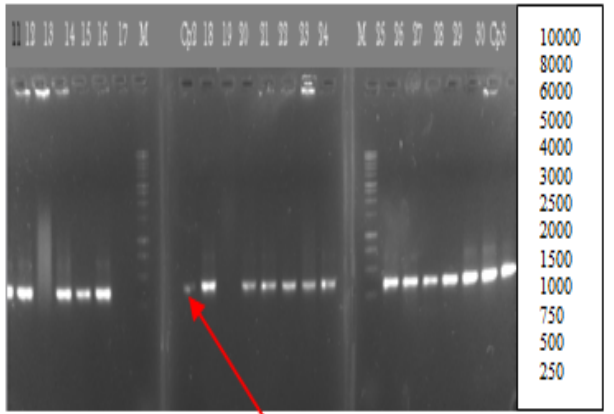

CP2 : 340 bp = l copy

c.VNTR MU / VNTR 19

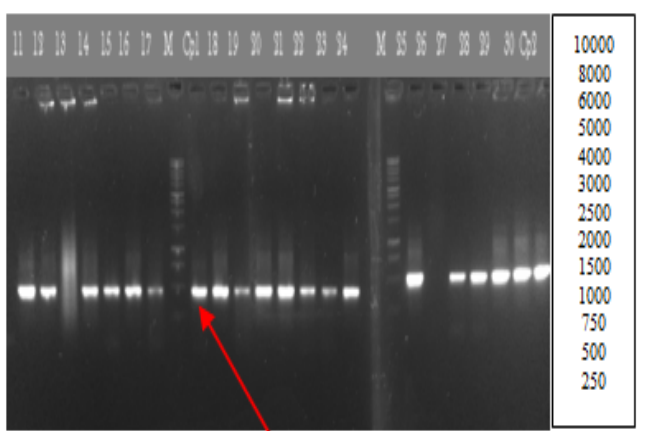

CPl: $500 \mathrm{bp}=3$ copies

B. VNTR MU / VNTR 6

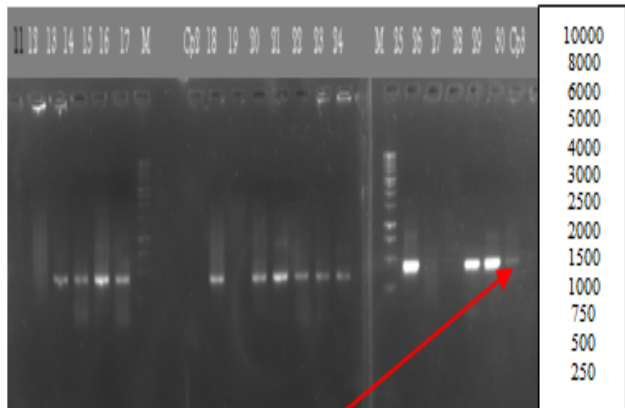

Réf: $423 \mathrm{bp}=2$ copies

D. VNTR MU / Locus ST-1

Figure 5. VNTR profiles defined from Miru-1, VNTR 6, VNTR 19 and ST-1 loci typing. 
Table 4. Geographical distribution of mycobacterial isolates and VNTR profiles

\begin{tabular}{|c|c|c|c|c|c|c|c|c|}
\hline \multirow{2}{*}{ Régions } & \multirow{2}{*}{ Isolats } & \multicolumn{2}{|c|}{ Séquences d'insertion } & \multicolumn{4}{|c|}{ Marqueurs MIRU-VNTR } & \multirow{2}{*}{$\begin{array}{c}\text { Profil } \\
\text { détermine }\end{array}$} \\
\hline & & IS2606 & IS2404 & Miru-1 & Locus 6 & Locus 19 & ST-1 & \\
\hline \multirow{5}{*}{$\begin{array}{l}\text { Tonkpi } \\
(n=9)\end{array}$} & CI-29 ; CI-37 & - & + & - & - & + & + & [0012] \\
\hline & $\begin{array}{l}\text { CI-11 ; CI-08 } \\
\text { CI-35 ; CI-28 }\end{array}$ & + & + & + & + & + & + & [3312] \\
\hline & CI-18 & + & + & + & + & - & + & [3302] \\
\hline & CI-36 & - & + & - & + & - & + & [0302] \\
\hline & CI-13 & - & + & - & - & - & - & [0000] \\
\hline \multirow{3}{*}{$\begin{array}{l}\text { Lôh Djiboua } \\
\qquad(\mathrm{n}=3)\end{array}$} & CI-09 & - & + & - & + & - & + & [0302] \\
\hline & CI-10 & + & + & + & + & + & + & [3312] \\
\hline & CI-27 & - & + & - & + & - & - & [0300] \\
\hline \multirow{4}{*}{$\begin{array}{c}\text { Mé } \\
(n=5)\end{array}$} & CI-06 & - & + & - & - & - & - & [0000] \\
\hline & CI-01 ; CI-02 & + & + & + & + & + & + & [3312] \\
\hline & CI-03 & + & + & + & + & - & + & [3302] \\
\hline & CI-04 & + & - & - & + & - & + & [0302] \\
\hline \multirow{3}{*}{$\begin{array}{l}\text { Gbêkê } \\
(n=6)\end{array}$} & CI-12 ; CI-22 & + & + & + & + & + & + & [3312] \\
\hline & $\begin{array}{l}\text { CI-23 ; CI-24 ; CI- } \\
31\end{array}$ & + & + & + & + & - & + & [3302] \\
\hline & CI-52 & - & + & - & + & - & + & [0302] \\
\hline \multirow{4}{*}{$\begin{array}{c}\text { Marahoué } \\
(\mathrm{n}=4)\end{array}$} & CI-39 & - & + & - & - & - & + & [0002] \\
\hline & CI-41 & + & + & + & + & - & + & [3302] \\
\hline & CI-42 & - & + & - & - & - & - & [0000] \\
\hline & CI-40 & + & + & + & + & + & + & [3312] \\
\hline \multirow[b]{2}{*}{$\begin{array}{l}\text { Bélier } \\
(\mathrm{n}=7)\end{array}$} & CI-25 ; CI-47 & + & - & - & + & - & - & [0300] \\
\hline & $\begin{array}{c}\text { CI-44 ; CI-45 } \\
\text { CI-46 ; CI-51 ; CI- } \\
53\end{array}$ & + & + & + & + & + & + & [3312] \\
\hline \multirow{5}{*}{$\begin{array}{l}\text { Agnéby-Tiassa } \\
(n=5)\end{array}$} & CI-13/21 & - & + & - & - & - & + & [0002] \\
\hline & CI-13/29 & - & + & - & - & - & - & [0000] \\
\hline & CI-36/16 & + & + & + & + & + & + & [3312] \\
\hline & CI-37/16 & + & + & - & + & - & + & [0302] \\
\hline & CI-64 & + & + & + & + & - & + & [3302] \\
\hline Sud-Comoé (n=3) & $\begin{array}{l}\text { CI-60 ; CI-55 ; CI- } \\
56\end{array}$ & + & + & + & + & + & + & [3312] \\
\hline \multirow{4}{*}{$\begin{array}{l}\text { Gôh } \\
(n=5)\end{array}$} & CI-72 & - & + & - & - & - & - & [0000] \\
\hline & CI-15/02 ; CI-101 & + & + & + & + & + & + & [3312] \\
\hline & CI-34/16 & + & + & - & + & - & + & [0302] \\
\hline & CI-153 & - & + & - & - & - & + & [0002] \\
\hline
\end{tabular}

M. ulcerans VNTR profile [3312]; M. ulcerans VNTR profile [3302] ;

M. ulcerans VNTR profile [0302]; 1 , VNTR profiles of others atypical mycobacteria

$72.3 \%$ of the isolates were carried the KR gene coding for the plasmid of virulence. 27 out of $30 \mathrm{M}$. ulcerans strains (90\%) were amplified the KR gene. $71.4 \%$ of isolates from genotype 3 and $50 \%$ isolates from genotype 5 possessed the virulence genes. But, the virulence factor genes were not found in the strains from genotype 4 (Figure 4).

The rate of MIRU-VNTR loci amplification varied from one locus to another. ST-1marker was the most amplified locus by $80.8 \%$ of the isolates (38/47) with an amplification size of 340 base pairs (bp) corresponding to two replicates (Figure 5D). VNTR 6 locus was amplified by $78.7 \%$ of the isolates (37/47) and by two reference controls at a length of $500 \mathrm{bp}$, corresponding to three replicates (Figure 5B). In the third position, Miru-1 locus was amplified by $61.7 \%$ of isolates (29/47) and by all 3 controls, with 3 tandem repeats at a length of 500 bp (Figure 5A). VNTR 19 was the least amplified locus by $51 \%$ of the isolates (24/47) according to the reference controls at a length of $340 \mathrm{bp}$, corresponding to one tandem repeat (Figure 5C).

Seven VNTR profiles were identified among the mycobacteria isolates (Table 4). Three different profiles were determined in $M$. ulcerans strains based on the number of amplified loci. In profile 1, all targeted VNTR loci were amplified with 3 copies of Miru-1 and VNTR 6, 1 copy of VNTR 19 and 2 copies of ST-1. This profile named [3312] was identified in $44.7 \%$ of isolates (21/47) and in $70 \%$ of $M$. ulcerans strains (21/30). In profile 2, the VNTR 19 locus was not amplified. That profile named [3302] consisted of $23.3 \%$ of $M$. ulcerans strains (7/30). In profile 3, the Miru-1 and VNTR 19 loci were not amplified, unlike the reference control [0302]. It was identified in $6.7 \%$ of $M$. ulcerans strains (2/30) and in $17.6 \%$ of the non-M. ulcerans mycobacteria isolates (3/17). Four profiles were only identified in non- $M$. ulcerans mycobacteria isolates. The profiles [0300] and [0002] were found in $6.4 \%$ of the isolates (3/47), where only the VNTR 6 and ST-1 loci were respectively amplified. The profile [0012] was identified in 4.3\% (2/47) of non-M. ulcerans mycobacteria isolates, where both VNTR 19 and ST-1 loci were simultaneously amplified. In $10.6 \%$ of the mycobacteria isolates (5/47), none targeted locus was amplified [0000]. 


\section{Discussion}

The rate of bacterial isolation was different according to the stage of the lesions. It was better when lesions were early (45.2\%) and the skin lesions were not subject to any antibiotic treatment. So the bacilli were more viable to grow easily on Löwenstein-Jensen medium. Conversely, the isolation rate was low when lesions were old, which had mostly undergone antibiotic treatments that destroyed or weakened the bacilli. In 2009, Ahoua and al. obtained an isolation rate of $22.1 \%$. However, she didn't refer to either the stage of the lesions or the antibiotic treatments received by the patients [17].

$8.5 \%$ of the strains were isolated from lesions after the end of antibiotic treatment according to WHO protocol. That treatment combining Rifampicin and Streptomycin was directly and daily administered by health workers. Mycobacteria isolation after 8 weeks treatment appeared abnormal and caused suspicions of strains that may have particular characteristics. Different phenotypes were identified among the isolates based on microbiological characters described. For the dominant phenotype (65.9\%), bacteriological and biochemical characteristics were identical to those of a $M$. ulcerans strain. $6.4 \%$ of the isolates expressed bacteriological and biochemical characteristics in favor of a M. marinum strain. For $27.6 \%$ of the isolates, these characteristics were associated with atypical mycobacteria, such as $M$. chelonae, $M$. smegmatis (Table 3).

The results of molecular typing targeting the insertion sequences IS6110, IS2606 and IS2404 showed differences between the isolates. Indeed, those targets were amplified in different proportions. IS2404 was amplified in the majority of the isolates (93.6\%), followed by IS6110 (74.5\%) and IS2606 (70.2\%), respectively. Five genotypes were identified according to the number of amplified sequences. A typical M. ulcerans strain possesses both IS2606 and IS2404 insertion sequences, as described by Stinear in 1999 [23]. In this study, only the strains of genotype 1 (53.2\%) and 2 (10.6\%) had microbiological, biochemical and molecular characteristics identical to those of a $M$. ulcerans strain. Thus in our context, M. ulcerans was responsible for $63.8 \%$ of skin lesions treated as "Buruli ulcer". The strains of the genotype 4 had IS6110 and IS2606 sequences and expressed microbiological and biochemical characteristics identical to those of a M. marinum strain (6.4\%). The strains of genotypes 3 and 5 (29.8\%) expressed microbiological, biochemical and molecular characteristics relating to various atypical mycobacteria, such as $M$. smegmatis and $M$. chelonae (Table 3). Because of the possession of IS2404 sequence, those pathogens were reported and treated as M. ulcerans strains. But they were not.

Geographical distribution of the isolates showed a different circulation of genotypes in the endemic areas of Côte d'Ivoire. M. ulcerans strains of genotype 1 were found everywhere patients were enrolled. In some localities of the Sud-Comoé region (Bonoua and Adiahou), the genotype 1 was the only one detected. Both localities are in agricultural areas drained by the Comoé River and numerous wetlands. M. ulcerans strains of genotype 2 were found in central (Gbêkê, Marahoué) and southern (Mé, Agnéby-Tiassa) regions of Côte d'Ivoire. The geographical distribution of genotypes 1 and 2 provided information on the circulation of $M$. ulcerans in endemic areas of Cote d'Ivoire. Other mycobacteria of genotypes 3 and 5 with IS2404 sequence were found in almost areas (7/9). Their presence could explain the high number of cases reported as "Buruli ulcer" in many localities, which may be mistaken for BU hyper-endemic areas. Some of those mycobacteria were isolated from patients after 8 weeks of well-conducted specific antibiotic therapy. This may explain the slow-healing lesions where Mycolactone and viable bacilli persisted after several weeks of antibiotic therapy [19,33,34].

The molecular typing targeting Miru-VNTR loci confirmed the variety of strains by showing the circulation of three VNTR profiles among M. ulcerans strains. Our results were similar to those found by Quinet and al. in 2017 [35]. The same loci were amplified in clinical samples and isolates from regions of Côte d'Ivoire in the same order of magnitude: ST-1 locus (57.4\%), MIRU-1 locus (42.3\%), VNTR 6 locus (30.8\%) and VNTR 19 locus (23.1\%). By contrast, Kakou [36] had amplified in 2015 those four loci but in proportions different from ours. In fact, she had found ST-1 in 85.7\%, MIRU-1 and VNTR 19 in $61.9 \%$ and VNTR 6 in 52\% of the samples tested, respectively. The MIRU-VNTR are variable repeat sequences with a new deletion or insertion in repetition loci. These different profiles would be evidence of a mutation in MIRU-VNTR loci in human strains. RFLP typing with better resolution showed differences between environmental mycobacterial strains and human $M$. ulcerans strains. Variations of MIRU-VNTR profiles could be a marker of adaptation of mycobacteria genotypes during the transition from the environment to humans. On the one hand, these studies confirmed a diversity of VNTR profiles between human M. ulcerans strains, and on the other hand between strains of atypical mycobacteria isolated from human skin lesions.

90\% (27/30) of $M$. ulcerans strains expressed virulence genes. The lack of amplification of the virulence genes could result from the loss of the plasmid by strains under the isolation or conservation conditions in the lab. In contrast, virulence genes were amplified in $63.6 \%$ of nonM. ulcerans strains possessing IS2404 sequence. This sequence is also carried by the plasmid pMUM001 as described by Stinear $[23,37,38]$. Its presence as well as that of the virulence genes could translate an acquisition of the plasmid by other mycobacteria living in the same ecosystem as M. ulcerans. Our results corroborated those reported by Vakou et al. [39] which isolated environmental fast-growing mycobacteria with both IS2404 and ketoreductase sequences. Transfer of the plasmid from $M$. ulcerans to environmental mycobacteria could confer virulence or enhance pathogenicity leading to necrotic skin lesions observed in patients. Conversely, virulence genes were not detected in the $M$. marinum strains identified in patients.

Molecular diagnosis of M. ulcerans infection based on IS2404 sequence and Ketoreductase gene detection may present limitations. The first limitation is that this method doesn't allow a differentiation between $M$. ulcerans strains and fast-growing or slow-growing mycobacteria, possessing IS2404 sequence or having acquired the plasmid pMUM001. Many cutaneous lesions probably caused by other mycobacteria could be attributed to M. ulcerans. The 
second limitation of this method is that it does not target IS2606 sequence found in M. marinum also involved in chronic skin ulcerations. The third limitation is that it could target environmental mycobacteria that contaminate skin ulcers. The best molecular diagnostic approach would be to target both insertion sequences IS2404-IS2606 and ketoreductase gene. On the one hand, this algorithm would discriminate between $M$. ulcerans and other environmental mycobacteria that also possess IS2404 sequence. On the other hand, it would determine the involvement of $M$. marinum in chronic skin lesions.

\section{Conclusion}

Mycobacterium ulcerans is an environmental mycobacterium that causes an endemic skin disease called "Buruli ulcer". Every year several health districts in Cote d'Ivoire report a large number of chronic ulcers, rightly or wrongly, as cases of Buruli ulcer.

Phenotypic and genomic characterization of strains isolated from Buruli ulcer suspected cases showed the presence of different mycobacteria species: M. ulcerans (63.8\%), M. marinum (6.4\%) and other atypical mycobacteria, such as $M$. chelonae and $M$. smegmatis (29.8\%). Most of M. ulcerans strains (90\%) and atypical mycobacterial strains (63.6\%) expressed genes encoding mycolactone bio synthesis, with the exception of $M$. marinum strains. Geographical distribution of strains showed that $M$. ulcerans co-circulated with other mycobacteria strains in the same ecosystems. Some of those non-M. ulcerans mycobacteria possessed insertion sequences (IS2404, IS2606) and virulence genes.

\section{Acknowledgements}

We are grateful to Pasteur Institute of Cote d'Ivoire, MAP International and American Leprosy Missions for the financial and technical support in the achievement of the samples collection and lab tests processing. We thank the Institute of Tropical Medicine of Anvers for the backing of the activities of Pasteur Institute's Molecular Biology Platform through the external evaluation tests of quality and the gift of reference DNA used in this study. We also thank the National Program against Buruli ulcer and his district partners for having eased the access to the patients.

\section{References}

[1] Grosset J., Boivert H., Truffot-Pernot C. (1989). Mycobactéries. In: Le Minor L, Véron M., ed. Bactériologie médicale. Paris: Flammarion; 1989: 965-1017.

[2] Marston B J, Diallo M O, Horsburgh C R Jr, Diomande I, Saki M Z, Kanga J M, Patrice G, Lipman H B, Ostroff S M, Good R C (1995) Emergence of Buruli ulcer disease in the Daloa region of Cote d'Ivoire. Am J Trop Med Hyg.; 52; 3: 219-224.

[3] Meyers W M, Connor D H, McCullough B, Bourland J, Moris R, Proos L (1974) Distribution of Mycobacterium ulcerans infections in Zaire, including the report of new foci. Ann Soc Belg Med Trop; 54: $147-157$

[4] OMS (2008). Ulcère de Buruli: rapport 2004-2008. Weekly epidemiological record, $\mathrm{N}^{\circ} 17,25$ April 2008.

[5] Perraudin M. L., Herrault A., Desbois J. C. (1980) Ulcère cutané à Mycobacterium ulcerans (ulcère de Buruli). Ann Péd; 27: 687-692.
[6] Faber W R, Bouda-Arias Pereira L M, Zeegelaar J.E, Kolk A H J, Fonteyne P- A, Toonstra J, Portaels F (2000). First reported case of Mycobacterium ulcerans infection in a patient from China. Trans Roy Soc Trop Med Hyg; 94: 277-279.

[7] Nakanaga K, Hoshino Y, Yotsu RR, Makino M, Ishii N (2011): Nineteen cases of Buruli ulcer diagnosed in Japan from 1980 to 2010. J Clin Microbiol; 49(11): 3829-36.

[8] Nakanaga K, Ishii N, Suzuki K, Tanigawa K, Goto M et al. (2007) "Mycobacterium ulcerans subsp. shinshuense" isolated from a skin ulcer lesion: identification based on 16S rRNA gene sequencing. J Clin Microbiol; 45; 11: 3840-3843.

[9] Yotsu RR, Nakanaga K, Hoshino Y, Suzuki K, Ishii N (2012). Buruli ulcer and current situation in Japan: a new emerging cutaneous Mycobacterium infection. J Dermatol; 39(7): 587-93.

[10] Anne-Caroline C, Tresse C, Casarottoa V, Nicolas B (2013). History, biology and chemistry of Mycobacterium ulcerans infections (Buruli ulcer disease). Nat. Prod. Rep. 30:1527-1567.

[11] Johnson P D, Azuolas J, Lavender C J, Wishart E, Stinear T P, Hayman J A, Brown L, Jenkin G A, Fyfe J A (2007). Mycobacterium ulcerans in mosquitoes captured during outbreak of Buruli ulcer, southeastern Australia. Emerg Infect Dis; 13; 11: 1653-1660.

[12] Mac C, Toh JC, Buckle G, Sis-Sons HA (1948). A new bacterial infection in man: clinical aspects. J. Pathol. Bacteriol. 60: 93-101.

[13] Dos Santos V M, Noronha F L, Vicentina E C, Lima C C (2007) Mycobacterium ulcerans infection in Brazil. Med J Aust; 187: 63-64.

[14] Guerra H, Palomino J C, Falconí E, Bravo F, Donaires N, Van Marck E, Portaels F (2008) Mycobacterium ulcerans Disease, Peru. Emerg Inf Dis; 14; 3: 373-377.

[15] McGann H, Stragier P, Portaels F, Gascoyne-Binzi D, Collyns T, Lucas S, Mawer D (2009) Buruli Ulcer in United Kingdom Tourist Returning from Latin America. Emerg Inf Dis; 15; 11: 1847-1859.

[16] OMS (2013). Recommandations pour la lutte contre l'ulcère de Buruli. Genève 25-27 mars 2013.

[17] Laurence A, Aka N, Euloge E, Samir B, Raymond N, Mireille D (2009). Risk factors for Buruli ulcer in Côte d'Ivoire: Results of a case-control study. African Journal of Biotechnology Vol. 8 (4), pp. 536-546, 18.

[18] Eddyani M, Fraga AG, Schmitt F, Uwizeye C, Fissette K, Johnson C, Aguiar J, Sopoh G, Barogui Y, Meyers WM, Pedrosa J, Portaels F (2009). Fine-needle aspiration, an efficient sampling technique for bacteriological diagnosis of non-ulcerative Buruli ulcer. J. Clin. Microbiol. 47(6): 1700-1704.

[19] Aka N'guetta, KAKOU-NGAZOA E. Solange, COULIBALY N. D., VAKOU Sabine, KOUADIO Kouamé, AOUSSI Serge, KADIO M. Constance, KOUAKOU Hélène, AKE Julien, N'GUESSAN Raymond and DOSSO Mireille (2015). "Virulence factors expressed by Mycobacterium ulcerans strains: Results of a descriptive study". African Journal of Microbiology Research, Vol. 9(28), pp. 1745-1751.

[20] Adalbert L, Mohammed A, Gisela B, Fadila B, David D, Lanfranco F, Knut F, Thomas F(2000). (Union) : Diagnostic de la tuberculose par examen microscopique direct des expectorations dans les pays à faibles revenus: Guide technique. Cinquième édition 2000, 60 pages.

[21] Guide de Bonne Exécution des Examens de Laboratoire (GBEA), $N^{\circ} 287$, du 11 décembre 1999, page 18441.

[22] Wilton S, Cousins D (1992). Detection and identification of multiple mycobacterial pathogens by DNA amplification in a single tube. PCR Methods Appl; 1: 269-273.

[23] Stinear T P, Ross B, Davis J, Marino L, Robins-Browne R, Oppedinaso F, Sievers A, Johnson P D (1999). Identification and characterization of IS2404 and IS2606: two distinct repeated sequences for detection of Mycobacterium ulcerans by PCR. J Clin Microbiol; 37: 1018-1023.

[24] Anthony Ablordey, Jean Swings, Christine Hubans, Karim Chemlal, Camille Locht, Françoise Portaels and Philip Supply (2005). Multilocus Variable-Number Tandem Repeat Typing of Mycobacterium ulcerans. Journal of clinical Microbiology, p. $1546-1551$.

[25] Stragier P, Ablordey A, Durnez L, Portaels F (2007) VNTR analysis differentiates Mycobacterium ulcerans and IS2404 positive mycobacteria. Syst Appl Microbiol; 30: 525-530. 
[26] Stragier P, Ablordey A, Bayonne L M, Lugor Y L, Sindani I S, Suykerbuyk P, Wabinga H, Meyers W M, Portaels F (2006) Heterogeneity among Mycobacterium ulcerans isolates from Africa. Emerg Infect Dis; 12: 844-847.

[27] Stragier P, Ablordey A, Meyers W M, Portaels F (2005) Genotyping Mycobacterium ulcerans and Mycobacterium marinum by using mycobacterial interspersed repetitive units. J. Bacteriol; 187: 1639-1647.

[28] Ehuié Pierre (2000). Diagnostic de l'ulcère de Buruli par la détection de l'IS2606: Une étude expérimentale à partir d'exsudats cutanés provenant de malades résidant dans la région des Lacs en 1998 en Côte d'Ivoire. Mémoire pour le Diplôme d'Ingénieurs des Techniques Sanitaires - Option Microbiologie, 95 p.

[29] Elise SN, Euloge E, Nguetta A, David CN, Bakary C, Mireille D (2011). Evaluation of real-time PCR for Mycobacterium ulcerans in endemic region in Côte d'Ivoire. African Journal of Microbiology Research. 5(16):2211-2216.

[30] Fyfe JA, Lavender CJ, Johnson PD, Globan M, Sievers A, Azuolas J, Stinear TP (2007). Development and application of two multiplex real-time PCR assays for the detection of Mycobacterium ulcerans in clinical and environmental samples. Appl. Environ. Microbiol. 73(15): 4733-4740.

[31] Rondini S, Mensah-Quainoo E, Troll H., Bodmer T, Pluschke, G. (2003). Development and application of real-time PCR assay for quantification of Mycobacterium ulcerans DNA. J Clin Microbiol; 41: 4231-4237.

[32] Ross B C, Johnson P D, Oppedisano F, Marino L, Sievers A, Stinear T, Hayman J A, Veitch M G, Robins-Browne R M (1997) Mycobacterium ulcerans in environmental samples during an outbreak of ulcerative diseases. Appl Environ Microbiol, 63: 41354138.

[33] Fred S Sarfo, Richard O Phillips, Jihui Zhang, Mohammed K Abass, Justice Abotsi, Yaw A Amoako, Yaw Adu-Sarkodie, Clive Robinson, Mark H Wansbrough-Jones (2014): Kinetics of mycolactone in human subcutaneous tissue during antibiotic therapy for Mycobacterium ulcerans disease. BMC Infect. Dis. 14: 202.
[34] Fred SS, Fabien LC, N'Guetta A, Richard OP, Yaw A, Ivo GB, Pascal L, Mireille D, Mark WJ, Romain VC, Laure GM, Caroline D (2011). Mycolactone Diffuses into the Peripheral Blood of Buruli Ulcer Patients - Implications for Diagnosis and Disease Monitoring. PLoS Negl. Trop. Dis. 5(7): e1237.

[35] Quinet G, Kakou-Ngazoa E S, Aka N, Vakou S, Coulibaly N D, Kouakou H, Sylla A, Faye-Kette H, Aoussi S and Dosso M (2017). Molecular Typing using PCR-RFLP Reveals Diversity of Environmental Mycobacteria Agent of Buruli Ulcer in Ivory Coast, Cote d'Ivoire (West Africa). Intern Med, an open access journal, Vol 7: 3.

[36] Kakou Ngazoa E. Solange, Coulibaly N. David, Aka Nguetta, Vakou Sabine, Aoussi Serge and Dosso Mireille (2015). Clonality of Mycobacterium ulcerans by Using VNTR-MIRU Typing in Ivory Coast (Côte d'Ivoire), West Africa. International Journal of Tropical Disease \& Health 7(4): 163-171.

[37] Stinear T P, Mve-Obiang A, Small P L, Frigui W, Pryor M J, Brosch R, Jenkin G A, Johnson P D R, Davies J K, Lee R E, Adusumilli S, Garnier T, Haydock S F, Leadlay P F, Cole S T ( 2004) Giant plasmid-encoded polyketide synthases produce the macrolide toxin of Mycobacterium ulcerans. Proc Natl Acad Sci U $S$ A, 101; 5: 1345-1349.

[38] Timothy PS, Melinda JP, Jessica LP, Stewart TC (2005). Functional analysis and annotation of the virulence plasmid pMUM001 from Mycobacterium ulcerans. Microbiology. 151: 683-692.

[39] Sabine N. Vakou, Julien Coulibaly-Kalpy, Solange E. KakouNgazoa, N`guetta Aka,N`golo D. Coulibaly, Mireille Dosso and Allico J. Djaman (2016). Microbiological and molecular characterization of environmental mycobacterium strains isolated from the Buruli ulcer endemic and non-endemic zones in Cote d'Ivoire. African Journal of Microbiology Research, Vol.10 (40), pp. 1703-1710.

[40] Oniangue NC (2000) Validation de l'amplification de la séquence d'insertion IS2404 pour le diagnostic de l'ulcère de Buruli en Côte d'Ivoire. Mémoire DEA de Biologie Humaine Tropicale. Option microbiologie. 2000, 66 pages. 